SHEP-12-01

DCP-12-01

\title{
Light charged Higgs bosons decaying to charm and bottom quarks in models with two or more Higgs doublets
}

\author{
A.G. Akeroyd* and S. Morettit \\ School of Physics and Astronomy, University of Southampton, \\ Highfield, Southampton SO17 1BJ, United Kingdom, and \\ Particle Physics Department, Rutherford Appleton Laboratory, \\ Chilton, Didcot, Oxon OX11 OQX, United Kingdom \\ J. Hernández-Sánchez \\ Facultad de Ciencias de la Electrónica, \\ Benemérita Universidad Autónoma de Puebla, \\ Apdo. Postal 542, 72570 Puebla, Puebla, México, \\ and Dual C-P Institute of High Energy Physics, Méxicd
}

\begin{abstract}
Searches for light charged Higgs bosons $\left(H^{ \pm}\right)$in the decay of top quarks, $t \rightarrow H^{ \pm} b$, are being carried out at the LHC and at the Tevatron. It is assumed that the dominant decay channels for such an $H^{ \pm}$state are either $H^{ \pm} \rightarrow \tau \nu$ or $H^{ \pm} \rightarrow c s$, and separate searches are performed with comparable sensitivity to the parameters $m_{H^{ \pm}}$and $\tan \beta$ of the scalar potential. The branching ratio for the decay $H^{ \pm} \rightarrow c b$ can be as large as $80 \%$ in the Aligned Two Higgs Doublet Model and in models with three or more Higgs doublets with natural flavour conservation, while satisfying the constraint from $b \rightarrow s \gamma$ for $m_{H^{ \pm}}<m_{t}$. Although the current search strategy for $H^{ \pm} \rightarrow c s$ is also sensitive to $H^{ \pm} \rightarrow c b$, a considerable gain in sensitivity could be obtained by tagging the $b$ quark from the decay $H^{ \pm} \rightarrow c b$. Such an analysis, which could be readily performed at the Tevatron and in the $7 \mathrm{TeV}$ and $8 \mathrm{TeV}$ runs of the LHC, would probe a parameter space of the fermionic couplings of $H^{ \pm}$in the above models which at present cannot be probed by experimental observables in flavour physics.
\end{abstract}

PACS numbers: 14.80.Fd, 12.60.Fr

${ }^{*}$ Electronic address: a.g.akeroyd@soton.ac.uk

${ }^{\dagger}$ Electronic address: s.moretti@soton.ac.uk

${ }^{\ddagger}$ Electronic address: jaimeh@ece.buap.mx 


\section{INTRODUCTION}

There is much ongoing experimental effort by the ATLAS and CMS collaborations at the CERN Large Hadron Collider (LHC) to search for the neutral Higgs boson $\left(h^{0}\right)$ of the Standard Model (SM) [1, 2]. This model of spontaneous symmetry breaking will be tested at the LHC over all of the theoretically preferred mass range, in an experimental programme which is expected to be completed by the end of the $8 \mathrm{TeV}$ run of the LHC. At present [3] there are only two regions for the mass for $h^{0}$ which have not been excluded at $95 \%$ c.l: i) a region of light mass, with $122 \mathrm{GeV}<m_{h^{0}}<128 \mathrm{GeV}$, and ii) a region of heavy mass, $m_{h^{0}}>600 \mathrm{GeV}$.

However, this simplest model of one fundamental scalar with a vacuum expectation value (vev) might not be nature's choice. There could be additional scalar fields which also contribute to the masses of the fermions and weak bosons, with a more complicated scalar potential which depends on several arbitrary parameters. Importantly, even in the event of no signal for a SM-like Higgs boson at the LHC, the search for scalar particles should continue in earnest due to the fact that a non-minimal Higgs sector can give rise to different experimental signatures, some of which are challenging to detect. Consequently, it will take much longer for the LHC to probe all of the parameter space of such models.

A commonly-studied extension of the Higgs sector of the SM is the "Two Higgs Doublet Model" (2HDM), which is composed of two Higgs isospin doublets [4] (this model has recently been reviewed in [5]). Notably, this structure is necessary in the Minimal Supersymmetric (SUSY) extension of the SM (called the "MSSM"). The extra Higgs doublet gives rise to a particle spectrum with multiple Higgs bosons; three are electrically neutral (two are CP-even, one is CP-odd) and two are electrically charged (denoted by $H^{ \pm}$). Flavour-changing neutral currents (FCNCs) mediated by scalars at tree level can be eliminated by requiring that the scalar interactions with the fermions are invariant under discrete symmetries ("natural flavour conservation", NFC) [6]. The discovery of a charged scalar $H^{ \pm}$would be unequivocal evidence of a non-minimal Higgs sector, and there have been many studies of the prospects of directly observing $H^{ \pm}$from a 2HDM or the MSSM at the Tevatron and LHC [7] (for reviews see [5, 8]) Moreover, the effect of $H^{ \pm}$on the decay rates of mesons (especially $B$ mesons) also plays a major role in constraining $m_{H^{ \pm}}$and the fermionic couplings of $H^{ \pm}$ [7], [9].

If $m_{H^{ \pm}}<m_{t}+m_{b}$, such particles would most copiously (though not exclusively [10]) be produced in the decays of top quarks via $t \rightarrow H^{ \pm} b[11]$. Searches in this channel have been performed by the Tevatron experiments, assuming the decay modes $H^{ \pm} \rightarrow c s$ and $H^{ \pm} \rightarrow \tau \nu$ [12, 13]. Since no signal has been observed, constraints are obtained on the parameter space of $\left[m_{H^{ \pm}}, \tan \beta\right]$, where $\tan \beta=v_{2} / v_{1}$ (i.e. the ratio of the vacuum expectation values of the two scalar doublets). Searches in these channels have now been carried out at the LHC: i) for $H^{ \pm} \rightarrow c s$ with $0.035 \mathrm{fb}^{-1}$ by ATLAS [14], and ii) for $H^{ \pm} \rightarrow \tau \nu$ with $4.8 \mathrm{fb}^{-1}$ by ATLAS [15] and with $1 \mathrm{fb}^{-1}$ by CMS [16]. These are the first searches for $H^{ \pm}$at this collider. The constraints on $\left[m_{H^{ \pm}}, \tan \beta\right]$ from the LHC searches for $t \rightarrow H^{ \pm} b$ are now superior to those obtained from the corresponding Tevatron searches.

The phenomenology of $H^{ \pm}$in models with three or more Higgs doublets (called MultiHiggs Doublet Models, MHDM), was first studied comprehensively in [17], with an emphasis on the constraints from low-energy processes (e.g. the decays of mesons). Although the phenomenology of $H^{ \pm}$at high-energy colliders in a MHDM and in a 2HDM has many similarities, the possibility of $m_{H^{ \pm}}<m_{t}$ together with an enhanced branching ratio (BR) 
for $H^{ \pm} \rightarrow c b$ would be a distinctive feature of the MHDM. This scenario, which was first mentioned in [17] and studied in more detail in [18 20], is of immediate interest for the ongoing searches for $t \rightarrow H^{ \pm} b$ with $H^{ \pm} \rightarrow c s$ by the LHC [14]. Although the current limits on $H^{ \pm} \rightarrow c s$ can be applied to the decay $H^{ \pm} \rightarrow c b$ (as discussed in [21] in the context of the Tevatron searches), a further improvement in sensitivity to $t \rightarrow H^{ \pm} b$ with $H^{ \pm} \rightarrow c b$ could be obtained by tagging the $b$ quark which originates from $H^{ \pm}[19,21,22]$. We will estimate the increase in sensitivity to $\mathrm{BR}\left(H^{ \pm} \rightarrow c b\right)$ and to the fermionic couplings of $H^{ \pm}$in this scenario.

Large values of $\mathrm{BR}\left(H^{ \pm} \rightarrow c b\right)$ are also possible in certain 2HDMs, such as the "flipped 2HDM" with NFC [18, 21, 23]. However, in this model one would expect $m_{H^{ \pm}}>m_{t}$ due to the constraint from $b \rightarrow s \gamma\left(m_{H^{ \pm}}>295 \mathrm{GeV}[24[26])\right.$, and thus $t \rightarrow H^{ \pm} b$ with $H^{ \pm} \rightarrow c b$ would not proceed unless there were additional New Physics beyond that of the 2HDM which contributed to $b \rightarrow s \gamma$, and weakened the constraint on $m_{H^{ \pm}}$. In the "Aligned Two Higgs Doublet Model" (A2HDM) [27] there are no FCNCs (as is the case in a 2HDM with NFC) due to an alignment of the Yukawa couplings. The phenomenology of $H^{ \pm}$in the A2HDM [28] is very similar to that of $H^{ \pm}$in a MHDM [30], and $m_{H^{ \pm}}<m_{t}$ in the A2HDM is also compatible with constraints from $b \rightarrow s \gamma$. Our numerical results for $\mathrm{BR}\left(H^{ \pm} \rightarrow c b\right)$ in a MHDM apply directly to the A2HDM. The 2HDM without NFC and without alignment also has a sizeable parameter space for a large $\mathrm{BR}\left(H^{ \pm} \rightarrow c b\right)$, and a detailed study can be found in [22].

Our work is organised as follows. In section II we describe the fermionic interactions of $H^{ \pm}$in the MHDM/A2HDM. In section III we quantify the parameter space for a large $\mathrm{BR}\left(H^{ \pm} \rightarrow c b\right)$ in the MHDM/A2HDM. In section IV we summarise the Tevatron/LHC searches for $t \rightarrow H^{ \pm} b$ with $H^{ \pm} \rightarrow c s$ and discuss how they could be optimised for $H^{ \pm} \rightarrow c b$. Section V contains our numerical results, with conclusions in section VI.

\section{II. $H^{ \pm}$IN MODELS WITH MORE THAN TWO HIGGS DOUBLETS AND IN THE ALIGNED 2HDM}

In a general 2HDM each fermion type (i.e. up-type quarks, down-type quarks and charged leptons) couples to both of the scalar doublets. This would lead to FCNCs which are mediated by the neutral scalars, and the magnitude of the associated Yukawa coupling is constrained by experimental data (especially meson-antimeson mixing and the decays of mesons). Such FCNCs can be suppressed by assuming that the flavour-changing Yukawa couplings are very small, which can be achieved by invoking a specific structure of the fermion mass matrices [31]. An alternative approach is to eliminate the FCNCs by requiring that the Lagrangian is invariant under a discrete symmetry, which is achieved if each species of fermion couples to at most one scalar doublet (NFC). This condition leads to four distinct types of 2HDMs which differ in their Yukawa couplings. These four models are called Model I, Model II, Lepton-specific and Flipped. ${ }^{1}$ Models I and II have received much phenomenological attention, while the study of the Lepton-specific and Flipped models has been revived recently [21, 23, 33, 34], with early studies in [18, 20, 35]. We now introduce the fermionic couplings of $H^{ \pm}$in a MHDM and the A2HDM, and discuss how these couplings

1 The Lepton-specific and Flipped models are referred to as IIA and IIB in [32], IV and III in [7], I' and II' in [17 20] and $X$ and $Y$ in [23]. 
differ from those in the above 2HDMs with NFC.

\section{A. The Multi-Higgs Doublet Model (MHDM)}

A MHDM is an extension of the $2 \mathrm{HDM}$ with $n$ scalar doublets, where $n \geq 3$. The suppression of FCNCs is obtained by imposing NFC. As in the 2HDM, the MHDM has the virtue of predicting $\rho=1$ at tree level, with finite higher-order corrections which depend on the mass splittings of the scalars. In the MHDM there are $n-1$ charged scalars, and a detailed study of the phenomenology of the lightest $H^{ \pm}$in such models was performed in [17], with the assumption that the other $H^{ \pm}$are much heavier. The interaction of the lightest $H^{ \pm}$in a MHDM with the fermions is described by the following Lagrangian:

$$
\mathcal{L}_{H^{ \pm}}=-\left\{\frac{\sqrt{2} V_{u d}}{v} \bar{u}\left(m_{d} X P_{R}+m_{u} Y P_{L}\right) d H^{+}+\frac{\sqrt{2} m_{e}}{v} Z \overline{\nu_{L}} \ell_{R} H^{+}+H . c .\right\}
$$

Here $u$ and $d$ denote up-type quarks and down-type quarks respectively (for all three generations); $V_{u d}$ is a CKM matrix element; $m_{u}, m_{d}$ and $m_{e}$ are the masses of the uptype quarks, down-type quarks and charged leptons respectively; $P_{L}$ and $P_{R}$ are chirality projection operators, and $v=246 \mathrm{GeV}$. In a $2 \mathrm{HDM}$ with natural flavour conservation, the couplings $X, Y$ and $Z$ are determined solely by $\tan \beta=v_{2} / v_{1}$. The values of $X, Y$ and $Z$ in the four versions of the 2HDM [7] are given in Table I. It is clear that $|X|,|Y|$ and $|Z|$ are simply related in the $2 \mathrm{HDM}$ e.g. one has $|X|=|Z|=1 /|Y|$ for the Type II structure.

\begin{tabular}{|c||c|c|c|}
\hline & $X$ & $Y$ & $Z$ \\
\hline Type I & $-\cot \beta$ & $\cot \beta$ & $-\cot \beta$ \\
Type II & $\tan \beta$ & $\cot \beta$ & $\tan \beta$ \\
Lepton-specific & $-\cot \beta$ & $\cot \beta$ & $\tan \beta$ \\
Flipped & $\tan \beta$ & $\cot \beta$ & $-\cot \beta$ \\
\hline
\end{tabular}

TABLE I: The couplings $X, Y$ and $Z$ in the Yukawa interactions of $H^{ \pm}$in the four versions of the $2 \mathrm{HDM}$ with natural flavour conservation.

In a MHDM the couplings $X, Y$ and $Z$ are arbitrary complex numbers, which are defined in terms of the $n \times n$ matrix $U$ which diagonalises the mass matrix of the charged scalars:

$$
X_{i}=\frac{U_{d i}}{U_{d 1}}, \quad Y_{i}=-\frac{U_{u i}}{U_{u 1}}, Z_{i}=\frac{U_{e i}}{U_{e 1}} .
$$

We follow the notation of [17] in which $i=1$ corresponds to the couplings of the charged Goldstone boson, and $i$ runs from 2 to $n$ for the physical charged scalars. The fermionic couplings of the lightest $H^{ \pm}$in a MHDM are taken to be $X_{2}, Y_{2}$ and $Z_{2}$. The subscripts $d, u, e$ take any integer value up to $n$, and specify which of the $n$ doublets couples to which fermion type e.g. for a Type II structure one sets $d=e=1$ and $u=2$, while for the "democratic" $3 \mathrm{HDM}$ (e.g. [30]) one has $d=1, u=2$ and $e=3$. In a $2 \mathrm{HDM}, U$ is a $2 \times 2$ matrix with 
elements given by $\sin \beta$ and $\cos \beta$ (i.e. one free parameter). In a $3 \mathrm{HDM}, U$ is a $3 \times 3$ matrix with four free parameters which can be taken as $\tan \beta=v_{u} / v_{d}, \tan \gamma=\sqrt{\left(v_{d}^{2}+v_{u}^{2}\right)} / v_{e}$, a mixing angle $\theta$ for the two $H^{ \pm}$, and a complex phase $\delta$. An explicit form of the matrix $U$ for the 3HDM is given in [30].

Due to the unitarity of the matrix $U$ one can derive the following identities [17]:

$$
\begin{aligned}
& \sum_{i=2}^{n} X_{i} Y_{i}^{*}=1(\text { for } d \neq u), \\
& \sum_{i=2}^{n} X_{i} Z_{i}^{*}=-1(\text { for } d \neq e), \\
& \sum_{i=2}^{n} Y_{i} Z_{i}^{*}=1(\text { for } u \neq e),
\end{aligned}
$$

and

$$
\sum_{i=2}^{n}\left|X_{i}\right|^{2}=\frac{v^{2}}{v_{d}^{2}}-1, \sum_{i=2}^{n}\left|Y_{i}\right|^{2}=\frac{v^{2}}{v_{u}^{2}}-1, \sum_{i=2}^{n}\left|Z_{i}\right|^{2}=\frac{v^{2}}{v_{e}^{2}}-1 .
$$

In a $2 \mathrm{HDM}$ these identities reduce to simple trigonometric relations involving $\tan \beta$. It is evident that the branching ratios of $H^{ \pm}$to fermions in the MHDM depend on the threedimensional parameter space of $X_{i}, Y_{i}$ and $Z_{i}$, in contrast to the case in the 2HDM where a single parameter $(\tan \beta)$ determines these three couplings. It is conventional to consider the phenomenology of the lightest $H^{ \pm}$, assuming that the other $H^{ \pm}$are heavier. One then drops the $i$ subscript on the couplings of the lightest $H^{ \pm}$and uses the Lagrangian in eq. (11).

Many experimental observables in flavour physics would receive a contribution from $H^{ \pm}$, and thus the magnitudes of $X, Y$ and $Z$ are constrained. Detailed studies have been performed in [17] (in the context of a MHDM) and more recently in [28] in the context of the A2HDM (see also 9] $)$. For $m_{H^{ \pm}}<m_{t}$ these constraints are roughly as follows: $|Y|<1$ from $Z \rightarrow b \bar{b}$ (assuming $|X|<50$ ), $|Z|<40$ from leptonic $\tau$ decays, and $|X Z|<1080$ from $B^{ \pm} \rightarrow \tau \nu$. In this work we will derive constraints on $|X|$ from $t \rightarrow H^{ \pm} b$.

A particularly important constraint on the mass and couplings of $H^{ \pm}$in a $2 \mathrm{HDM} / \mathrm{MHDM}$ is the decay $b \rightarrow s \gamma[24$, 25], which has been measured to be in agreement with the SM prediction. It is the combination of couplings $X Y^{*}$ and $|Y|^{2}$ which enters the decay rate for $b \rightarrow s \gamma$ (the contribution from $|X|^{2}$ can be neglected). Since $X Y^{*}=1$ in Model II and the flipped $2 \mathrm{HDM}$ (i.e. $d \neq u$ ), the stringent bound $m_{H^{ \pm}}>295 \mathrm{GeV}$ at $95 \%$ c.l can be derived for all values of $\tan \beta[26$. In contrast, in the MHDM with $d \neq u$, the combination $X Y^{*}$ is only weakly constrained by the sum rule in eq. (3), and can be negative. For $d=u$ (e.g. Model I and the leptonic-specific 2HDM) the constraint in eq. (3) does not apply. Therefore a light $H^{ \pm}$(i.e. $m_{H^{ \pm}}<m_{t}$ ) being compatible with $b \rightarrow s \gamma$ is still a possibility. Recent studies of the bounds on $H^{ \pm}$of the MHDM from $b \rightarrow s \gamma$ [28, 29] derive the following approximate $2 \sigma$ intervals for the real part of $X Y^{*}$ with $m_{H^{ \pm}}=100 \mathrm{GeV}$ :

$$
-1.1<\operatorname{Re} X Y^{*}<0.7
$$

In deriving this constraint it is assumed that $|Y|$ is not so big (e.g. $|Y|<1$, which is required from other low-energy processes such as $Z \rightarrow b \bar{b}$ ). There is also a constraint on the imaginary part of $X Y^{*}$ from a different process $\left(\operatorname{Im} X Y^{*}<0.1\right.$ ), but for simplicity we will consider $X$ and $Y$ to be real. 


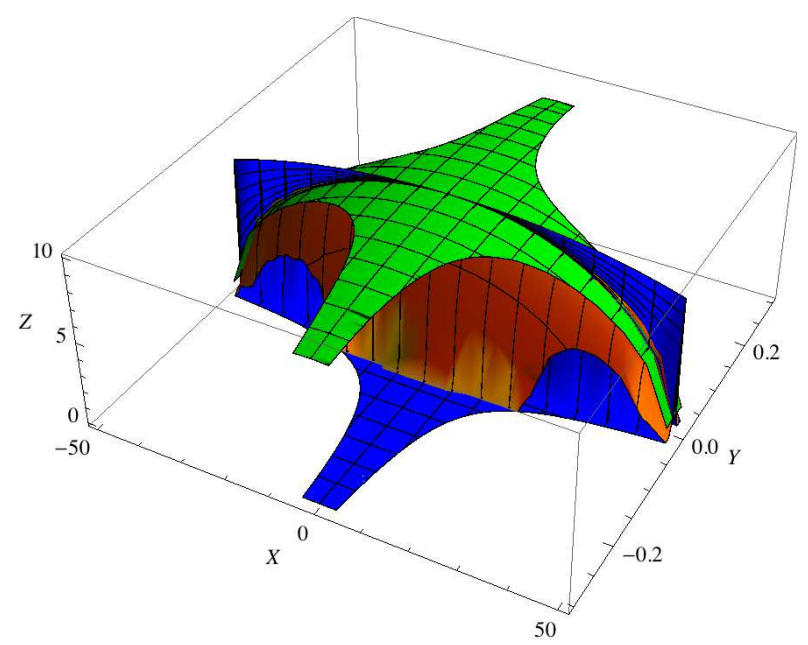

FIG. 1: The region of the $[X, Y, Z]$ plane allowed by the unitarity constraint of eq. (8). The constraint from $b \rightarrow s \gamma$ is shown as $\left|X Y^{*}\right|<1.1$ for $\operatorname{Re}\left(X Y^{*}\right)<0$, and $\left|X Y^{*}\right|<0.7$ for $\operatorname{Re}\left(X Y^{*}\right)>$ 0 .

In addition to the above phenomenological constraints, there are constraints on $X, Y$ and $Z$ from the unitarity of the matrix $U$ for the democratic 3HDM, which were studied in [17, 30]. There is a non-trivial relationship on the couplings of the lightest $H^{ \pm}$given as:

$$
\left|X_{2}\right|^{2}\left|U_{1 d}^{2}\right|+\left|Y_{2}\right|^{2}\left|U_{1 u}^{2}\right|+\left|Z_{2}\right|^{2}\left|U_{1 e}^{2}\right|=1
$$

This constraint ensures that the magnitudes of $X_{2}, Y_{2}$ and $Z_{2}$ cannot all be simultaneously less than one, or all be simultaneously greater than one. This is due to the fact that all three vacuum expectation values $\left(v_{d}, v_{u}, v_{e}\right)$ cannot be simultaneously large or small.

In our numerical analysis we will always take $|Y|<0.8$ (as discussed above), and so the requirement that all three couplings cannot be simultaneously greater than unity is automatically satisfied. We will be concerned with the parameter space of $|X|>>|Y|,|Z|$ (which corresponds to large $\tan \beta$ and moderate/small $\tan \gamma$ ), a choice which satisfies the requirement that all three couplings cannot be simultaneously less than unity. In Fig. (8) we show the region in the plane $[X, Y, Z]$ allowed by the unitarity constraint in eq. (8), imposing $|Y|<0.3$ and the constraint on $X Y^{*}$ in eq. (77). It can be seen that there is no parameter space where both of $|X|$ and $|Z|$ are less than unity, and the parameter of interest to us (i.e. $|X|>>|Y|,|Z|)$ is fully compatible with the unitarity constraint in eq. (8). This constraint on the couplings is removed in a $4 \mathrm{HDM}$ due to the presence of a fourth vacuum expectation value.

\section{B. The Aligned 2HDM (A2HDM)}

The A2HDM is a $2 \mathrm{HDM}$ in which NFC is not imposed [27], and both scalar doublets $\left(\Phi_{1}\right.$ and $\Phi_{2}$ ) couple to all types of fermions. Tree-level FCNCs are eliminated by imposing an alignment of the Yukawa couplings of $\Phi_{1}$ and $\Phi_{2}$. The interaction of $H^{ \pm}$with the fermions in the A2HDM can be written in the same way as eq. (1), but the couplings $X, Y$, and $Z$ are determined by five parameters (instead of the four parameters in the democratic 3HDM) and the unitarity constraint of eq. (8) does not apply. Apart from these two differences 
(which were discussed in [30]), the phenomenology of $H^{ \pm}$in the democratic 3HDM and the A2HDM is essentially the same, and our numerical results will apply equally to both models. In particular, the magnitudes of $X, Y$ and $Z$ determine the BRs of $H^{ \pm}$. In the A2HDM the extra free parameter can be taken to be a phase in the coupling $Y$, and such a phase does not have an effect on the BRs of $H^{ \pm}$. Moroever, we will be concerned with the parameter space of $|X|>>|Y|,|Z|$, which is compatible with the unitarity constraint in a 3HDM. Hereafter, when the text refers to " $H^{ \pm}$of the MHDM", the implicit meaning is for an $H^{ \pm}$ of a 3HDM, the A2HDM, and for a MHDM with more than three scalar doublets.

\section{A LARGE BR( $\left(H^{ \pm} \rightarrow c b\right)$ IN THE MHDM AND A2HDM}

In a MHDM and in the A2HDM the expressions for the partial widths of the decay modes of $H^{ \pm}$are:

$$
\begin{gathered}
\Gamma\left(H^{ \pm} \rightarrow \ell^{ \pm} \nu\right)=\frac{G_{F} m_{H^{ \pm}} m_{\ell}^{2}|Z|^{2}}{4 \pi \sqrt{2}} \\
\Gamma\left(H^{ \pm} \rightarrow u d\right)=\frac{3 G_{F} m_{H^{ \pm}}\left(m_{d}^{2}|X|^{2}+m_{u}^{2}|Y|^{2}\right)}{4 \pi \sqrt{2}}
\end{gathered}
$$

In $\Gamma\left(H^{ \pm} \rightarrow u d\right)$ the running quark masses should be evaluated at the scale of $m_{H^{ \pm}}$, and there are QCD vertex corrections which multiply the above partial widths by $\left(1+17 \alpha_{s} /(3 \pi)\right)$. In the $2 \mathrm{HDM}$ the parameter $\tan \beta$ determines the magnitude of the partial widths. The branching ratios are well known, and for the case of interest of $m_{H^{ \pm}}<m_{t}$ one finds that the dominant decay mode is either $H^{ \pm} \rightarrow c s$ or $H^{ \pm} \rightarrow \tau \nu$, depending on the value of $\tan \beta$. In model I the $\mathrm{BRs}$ are independent of $\tan \beta$, and $\mathrm{BR}\left(H^{ \pm} \rightarrow \tau \nu\right)$ is about twice that of $\mathrm{BR}\left(H^{ \pm} \rightarrow c s\right)$.

The magnitude of $\mathrm{BR}\left(H^{ \pm} \rightarrow c b\right)$ is always less than a few percent in three (Models I, II and lepton-specific) of the four versions of the $2 \mathrm{HDM}$ with NFC, since the decay rate is suppressed by the small CKM element $V_{c b}\left(\ll V_{c s}\right)$. In contrast, a sizeable $\mathrm{BR}\left(H^{ \pm} \rightarrow c b\right)$ can be obtained in the flipped $2 \mathrm{HDM}$ for $\tan \beta>3$. This possibility was not stated explicitly in [7] when the flipped $2 \mathrm{HDM}$ was discussed. The first explicit mention of a large $\mathrm{BR}\left(H^{ \pm} \rightarrow\right.$ $c b)$ seems to have been in [17], and a quantitative study followed soon afterwards in [18]. As discussed in section II, the condition $m_{H^{ \pm}}<m_{t}$ in the flipped 2HDM would require additional New Physics in order to avoid the constraint on $m_{H^{ \pm}}$from $b \rightarrow s \gamma$, while this is not the case in the MHDM.

\section{A. The dominance of $\mathbf{B R}\left(H^{ \pm} \rightarrow c b\right)$ for $|X|>>|Y|,|Z|$}

A distinctive signal of $H^{ \pm}$from a MHDM for $m_{H^{ \pm}}<m_{t}$ would be a sizeable branching ratio for $H^{ \pm} \rightarrow c b$. For $m_{H^{ \pm}}<m_{t}$, the scenario of $|X|>>|Y|,|Z|$ in a MHDM gives rise to a "leptophobic" $H^{ \pm}$with $\mathrm{BR}\left(H^{ \pm} \rightarrow c s\right)+\mathrm{BR}\left(H^{ \pm} \rightarrow c b\right) \sim 100 \%$. Consequently, $\mathrm{BR}\left(H^{ \pm} \rightarrow \tau \nu\right)$ is negligible $(<<1 \%)$. The other decays of $H^{ \pm}$to quarks are subdominant, with $\operatorname{BR}\left(H^{ \pm} \rightarrow u s\right) \sim 1 \%$ and $\operatorname{BR}\left(H^{ \pm} \rightarrow t^{*} b\right)$ only becomes sizeable for $m_{H^{ \pm}} \sim m_{t}$, as can be seen in the numerical analysis in [21] in the flipped 2HDM. Note that the case of $|X|>>$ $|Y|,|Z|$ is obtained in the flipped $2 \mathrm{HDM}$ for $\tan \beta>3$, because $|X|=\tan \beta=1 /|Y|=1 /|Z|$ in this model. 
In the scenario of $|X|>>|Y|,|Z|$ the ratio of the two dominant decays, $\operatorname{BR}\left(H^{ \pm} \rightarrow c b\right)$ and $\mathrm{BR}\left(H^{ \pm} \rightarrow c s\right)$, approaches a constant value, which is given as follows:

$$
\frac{\mathrm{BR}\left(H^{ \pm} \rightarrow c b\right)}{\mathrm{BR}\left(H^{ \pm} \rightarrow c s\right)}=R_{b s} \sim \frac{\left|V_{c b}\right|^{2} m_{b}^{2}}{\left|V_{c s}\right|^{2} m_{s}^{2}}
$$

The CKM elements are well measured, with $V_{c b} \sim 0.04$ (a direct measurement) and $V_{c s} \sim$ 0.97 (from the assumption that the CKM matrix is unitary). The running quark masses $m_{s}$ and $m_{b}$ should be evaluated at the scale $Q=m_{H^{ \pm}}$, and this constitutes the main uncertainty in the ratio $R_{b s}$. There is relatively little uncertainty for $m_{b}$, with $m_{b}(Q=100 \mathrm{GeV}) \sim 3$ $\mathrm{GeV}$. There is more uncertainty in the value of $m_{s}$, although in recent years there has been much progress in lattice calculations of $m_{s}$, and an average of six distinct unquenched calculations [36] gives $m_{s}=93.4 \pm 1.1 \mathrm{MeV}$ [37] at the scale of $Q=2 \mathrm{GeV}$. A more conservative average of these six calculations, $m_{s}=94 \pm 3 \mathrm{MeV}$, is given in [38]. In [39] the currently preferred range at $Q=2 \mathrm{GeV}$ is given as $80 \mathrm{GeV}<m_{s}<130 \mathrm{MeV}$. Using $m_{s}=93 \mathrm{MeV}$ at the scale of $Q=2 \mathrm{GeV}$ (i.e. roughly the central value of the lattice averages in [37, 38]) one obtains $m_{s}(Q=100 \mathrm{GeV}) \sim 55 \mathrm{MeV}$. Taking $m_{s}=80 \mathrm{MeV}$ and $m_{s}=130 \mathrm{MeV}$ at $Q=2 \mathrm{GeV}$ one obtains $m_{s} \sim 48 \mathrm{MeV}$ and $m_{s} \sim 78 \mathrm{MeV}$ respectively at $Q=100 \mathrm{GeV}$.

Smaller values of $m_{s}$ will give a larger $\operatorname{BR}\left(H^{ \pm} \rightarrow c b\right)$, as can be seen from eq. (11). Note that the value $m_{s}=55 \mathrm{MeV}$ is significantly smaller than the typical values $m_{s} \sim 150 \rightarrow 200$ $\mathrm{MeV}$ which were often used in Higgs phenomenology in the past two decades. We emphasise that the scenario of $|X|>>|Y|,|Z|$ with $m_{H^{ \pm}}<m_{t}$ has a unique feature that the magnitude of $m_{s}$ is crucial for determining the relative magnitude of the two dominant decay channels of $H^{ \pm}$. This is not the case for most other non-minimal Higgs sectors with $H^{ \pm}$that are commonly studied in the literature.

In [18] the magnitude of $\mathrm{BR}\left(H^{ \pm} \rightarrow c b\right)$ in the MHDM was studied in the plane of $|X|$ and $|Y|$, for $|Z|=0$ and 0.5 , taking $m_{s}=0.18 \mathrm{GeV}$ and $m_{b}=5 \mathrm{GeV}$. With these quark masses the maximum value is $R_{b s}=1.23$, which corresponds to $\operatorname{BR}\left(H^{ \pm} \rightarrow c b\right) \sim 55 \%$. However, the values of $m_{s}=0.18 \mathrm{GeV}$ and $m_{b}=5 \mathrm{GeV}$ are not realistic (as was subsequently noted in [20]), and two recent papers [21, 23] have updated the magnitude of $R_{b s}$ in the flipped 2HDM using realistic running quark masses at the scale of $m_{H^{ \pm}}$. In [21], it appears that $m_{s}=0.080$ $\mathrm{GeV}$ at the scale of $m_{H^{ \pm}}$was used, which gives $\mathrm{BR}\left(H^{ \pm} \rightarrow c b\right) \sim 70 \%$, in agreement with our results. In [23], $m_{s}=0.077 \mathrm{GeV}$ at the scale of $m_{H^{ \pm}}$was used, with a maximum value for $\operatorname{BR}\left(H^{ \pm} \rightarrow c b\right)$ of $\sim 70 \%$. We note that none of these papers used the precise average $m_{s}=93.4 \pm 1.1 \mathrm{MeV}$ [37] of the lattice calculations, which gives $m_{s} \sim 55 \mathrm{MeV}$ at the scale of $m_{H^{ \pm}}$. This smaller value of $m_{s}$ leads to a maximum value of $\operatorname{BR}\left(H^{ \pm} \rightarrow c b\right)$ which is larger than that given in [18, 21, 23], as discussed below.

We now study the magnitude of $H^{ \pm} \rightarrow c b$ as a function of the couplings $X, Y, Z$. In Fig. (2a) we update the numerical study of [18] for $\mathrm{BR}\left(H^{ \pm} \rightarrow c b\right)$ in the plane $[X, Y]$ in a MHDM with $|Z|=0.1$, using $m_{s}=0.055 \mathrm{GeV}$ and $m_{b}=2.95 \mathrm{GeV}$ at the scale of $m_{H^{ \pm}}=120$ $\mathrm{GeV}$. With these values for the quark masses the maximum value is $\operatorname{BR}\left(H^{ \pm} \rightarrow c b\right) \sim 81 \%$ i.e. a significantly larger value than $\mathrm{BR}\left(H^{ \pm} \rightarrow c b\right) \sim 55 \%$ in [18]. Taking a lower value of $m_{s}=0.08 \mathrm{GeV}$ one has $\mathrm{BR}\left(H^{ \pm} \rightarrow c b\right) \sim 69 \%$, and for $m_{s}=0.048 \mathrm{GeV}$ one has $\mathrm{BR}\left(H^{ \pm} \rightarrow c b\right) \sim 86 \%$. In Fig. (2a) we also display the bound from $b \rightarrow s \gamma$ (for $m_{H^{ \pm}}=100$ $\mathrm{GeV}$ ), which is $|X Y|<1.1$ for $X Y^{*}$ being real and negative, and $|X Y|<0.7$ for $X Y^{*}$ being real and positive. The parameter space for $\mathrm{BR}\left(H^{ \pm} \rightarrow c b\right)>60 \%$ roughly corresponds to $|X|>1$ and $|Y|<0.25$ for $|X Y|<0.7$. In Fig. (2b) and Fig. (2r) we show $\mathrm{BR}\left(H^{ \pm} \rightarrow c s\right)$ and 
$\mathrm{BR}\left(H^{ \pm} \rightarrow \tau \nu\right)$ respectively. As expected, $\mathrm{BR}\left(H^{ \pm} \rightarrow c s\right)$ is maximised for $|Y|>>|X|,|Z|$ while $\operatorname{BR}\left(H^{ \pm} \rightarrow \tau \nu\right)$ is maximised for $|Z|>>|X|,|Y|$. In Fig. (3) we show contours of $\mathrm{BR}\left(H^{ \pm} \rightarrow c b\right)$ in the plane $[X, Z]$ for $m_{H^{ \pm}}=120 \mathrm{GeV}$ and $|Y|=0.05$. For this value of $|Y|$ the constraint from $b \rightarrow s \gamma$ is always satisfied for the displayed range of $|X|<20$. One can see that the largest values of $\operatorname{BR}\left(H^{ \pm} \rightarrow c b\right)$ arise for $|Z|<2$.

\section{B. The decay $H^{ \pm} \rightarrow A^{0} W^{*}$ for $m_{A^{0}}<m_{H^{ \pm}}$}

The above discussion has assumed that $H^{ \pm}$cannot decay into other scalars. We now briefly discuss the impact of the decay channel $H^{ \pm} \rightarrow A^{0} W^{*}$, which has been studied in the 2HDM (Type II) in [40] and in other 2HDMs with small $|X|,|Y|$ and $|Z|$ in [20], with direct searches at LEP (assuming $A^{0} \rightarrow b \bar{b}$ ) performed in [41]. In a general non-SUSY 2HDM the masses of the scalars can be taken as free parameters. This is in contrast to the MSSM in which one expects $m_{H^{ \pm}} \sim m_{A^{0}}$ in most of the parameter space. The scenarios of $m_{A^{0}}<m_{H^{ \pm}}$and $m_{A^{0}}>m_{H^{ \pm}}$are both possible in a 2HDM, but large mass splittings among the scalars lead to sizeable contributions to electroweak precision observables [42], which are parametrised by the $S, T$ and $U$ parameters [43]. The case of exact degeneracy $\left(m_{A^{0}}=m_{H^{0}}=m_{H^{ \pm}}\right)$leads to values of $S, T$ and $U$ which are almost identical to those of the SM. A recent analysis in a $2 \mathrm{HDM}$ [44] sets $m_{H^{0}}=m_{A^{0}}$ and $\sin (\beta-\alpha)=1$, and studies the maximum value of the mass splitting $\Delta m=m_{A^{0}}-m_{H^{ \pm}}$(for earlier studies see [45]). For $m_{A^{0}}=100 \mathrm{GeV}$ the range $-70 \mathrm{GeV}<\Delta m<20 \mathrm{GeV}$ is allowed, which corresponds to $80 \mathrm{GeV}<m_{H^{ \pm}}<170 \mathrm{GeV}$. For $m_{A^{0}}=150 \mathrm{GeV}$ the allowed range is $-70 \mathrm{GeV}<\Delta m<70 \mathrm{GeV}$ which corresponds to $80 \mathrm{GeV}<m_{H^{ \pm}}<220 \mathrm{GeV}$. Consequently, sizeable mass splittings (of either sign) of the scalars are possible. Analogous studies in a MHDM have been performed in [46], with similar conclusions.

If $m_{A^{0}}<m_{H^{ \pm}}$then the decay channel $H^{ \pm} \rightarrow A^{0} W^{*}$ can compete with the above decays of $H^{ \pm}$to fermions, because the coupling $H^{ \pm} A^{0} W$ is not suppressed by any small parameter. In Fig. (4a) we show contours of $\operatorname{BR}\left(H^{ \pm} \rightarrow A^{0} W^{*}\right)$ in the plane $[X, Y]$ with $|Z|=0.1$, $m_{A^{0}}=80 \mathrm{GeV}$ and $m_{H^{ \pm}}=120 \mathrm{GeV}$. The contours are essentially vertical in the parameter space of interest (i.e. $|Y|<0.5$ and $|X|>>1$ ) because the contribution of the term $m_{c}^{2}|Y|^{2}$ to the decay widths of $H^{ \pm}$to fermions is small. Comparing Fig. (4a) and Fig. (2a) one can see that for $|X| \sim 5$ both $\mathrm{BR}\left(H^{ \pm} \rightarrow A^{0} W^{*}\right)$ and $\mathrm{BR}\left(H^{ \pm} \rightarrow c b\right)$ are dominant, with roughly equal BRs. For smaller $m_{A^{0}}$ (e.g. $<80 \mathrm{GeV}$ ) the contour of $\operatorname{BR}\left(H^{ \pm} \rightarrow A^{0} W^{*}\right)=50 \%$ would move to higher values of $|X|$. Since the dominant decay of $A^{0}$ is expected to be $A^{0} \rightarrow b \bar{b}$, the detection prospects in this channel should also be promising because there would be more $b$ quarks from $t \rightarrow H^{ \pm} b, H^{ \pm} \rightarrow A^{0} W^{*}, A^{0} \rightarrow b \bar{b}$ than from $t \rightarrow H^{ \pm} b$ with $H^{ \pm} \rightarrow c b$. We note that there has been a search by the Tevatron for the channel $t \rightarrow H^{ \pm} b, H^{ \pm} \rightarrow A^{0} W^{*}$, $A^{0} \rightarrow \tau^{+} \tau^{-}$[47], for the case of $m_{A^{0}}<2 m_{b}$ where $A^{0} \rightarrow b \bar{b}$ is not possible [48].

At present there is much speculation about an excess of events around a mass of $125 \mathrm{GeV}$ in the search for the SM Higgs boson [3]. An interpretation of these events as originating from the process $g g \rightarrow A^{0} \rightarrow \gamma \gamma$ has been suggested in [49]. In Fig. (44) we set $m_{A^{0}}=125$ $\mathrm{GeV}$ and $m_{H^{ \pm}}=150 \mathrm{GeV}$. Since the mass splitting between $H^{ \pm}$and $A^{0}$ is less than in Fig. (4a), the contours move to lower values of $|X|$, but $\operatorname{BR}\left(H^{ \pm} \rightarrow A^{0} W^{*}\right)=50 \%$ is still possible for $|X|<2$. We note that if the excess of events at $125 \mathrm{GeV}$ is attributed to a SMlike Higgs, then in the context of a $2 \mathrm{HDM}$ a candidate would be the lightest CP-even Higgs $h^{0}$ with a coupling to vector bosons of SM strength (recent studies of this possibility can be found in [50]). This scenario would correspond to $\sin (\beta-\alpha) \sim 1$ in a 2 HDM, and therefore 

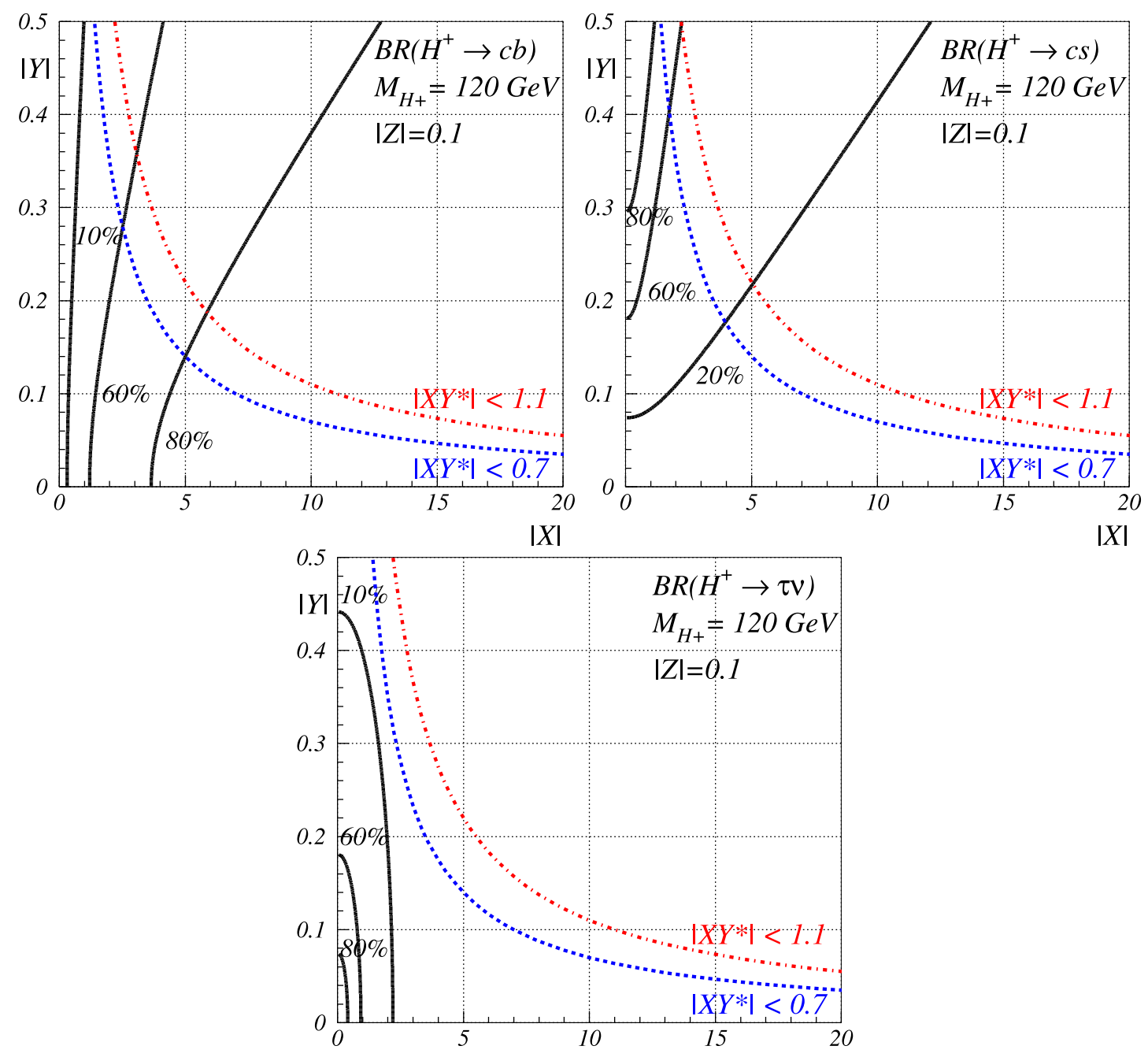

FIG. 2: Contours of $\mathrm{BR}\left(H^{ \pm} \rightarrow c b\right), \mathrm{BR}\left(H^{ \pm} \rightarrow c s\right)$ and $\mathrm{BR}\left(H^{ \pm} \stackrel{|X|}{\rightarrow} \tau \nu\right)$ in the plane $[X, Y]$ with $|Z|=0.1$. The constraint from $b \rightarrow s \gamma$ is shown as $\left|X Y^{*}\right|<1.1$ for $\operatorname{Re}\left(X Y^{*}\right)<0$, and $\left|X Y^{*}\right|<0.7$ for $\operatorname{Re}\left(X Y^{*}\right)>0$. We take $m_{s}\left(Q=m_{H^{ \pm}}\right)=0.055 \mathrm{GeV}$ and $m_{H^{ \pm}}=120 \mathrm{GeV}$.

the coupling $H^{ \pm} h^{0} W$ (with a magnitude $\sim \cos (\beta-\alpha)$ in a $2 \mathrm{HDM}$ ) would be close to zero. Hence the decay $H^{ \pm} \rightarrow h^{0} W^{*}$ would be suppressed by this small coupling, as well as by the virtuality of $W^{*}$. Several recent studies [51, 52] fit the current data in all the Higgs search channels to the case of a neutral Higgs boson with arbitrary couplings. A SM-like Higgs boson gives a good fit to the data, although a slight preference for non-SM like couplings is emphasised in [52]. If the excess of events at $125 \mathrm{GeV}$ turns out to be genuine and is well described by a non-SM like Higgs boson of a $2 \mathrm{HDM}$ with a value of $\sin (\beta-\alpha)$ which is significantly less than unity, then $\operatorname{BR}\left(H^{ \pm} \rightarrow h^{0} W^{*}\right)$ could be sizeable, with a magnitude given by Fig. (4b) after scaling by $\cos ^{2}(\beta-\alpha)$. 


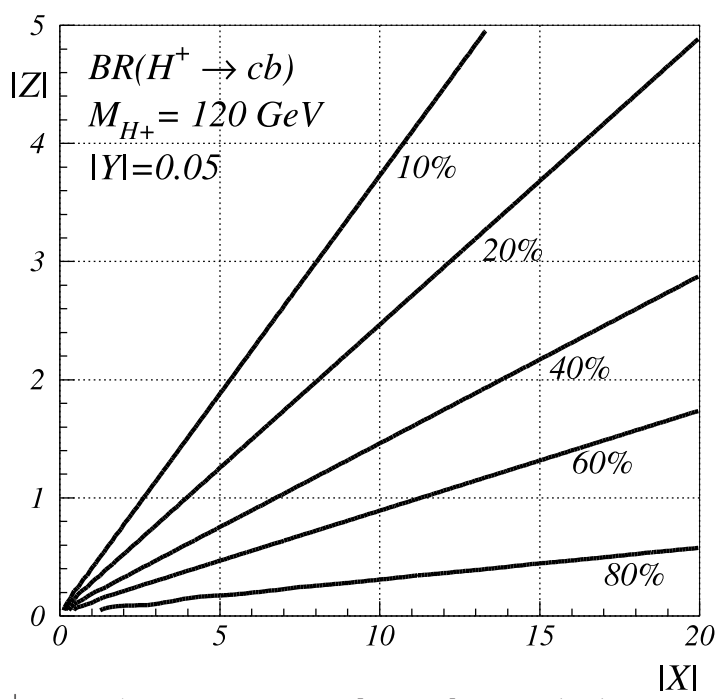

FIG. 3: Contours of $\operatorname{BR}\left(H^{ \pm} \rightarrow c b\right)$ in the plane $[X, Z]$ with $|Y|=0.05$. We take $m_{s}\left(Q=m_{H^{ \pm}}\right)=$ $0.055 \mathrm{GeV}$ and $m_{H^{ \pm}}=120 \mathrm{GeV}$.
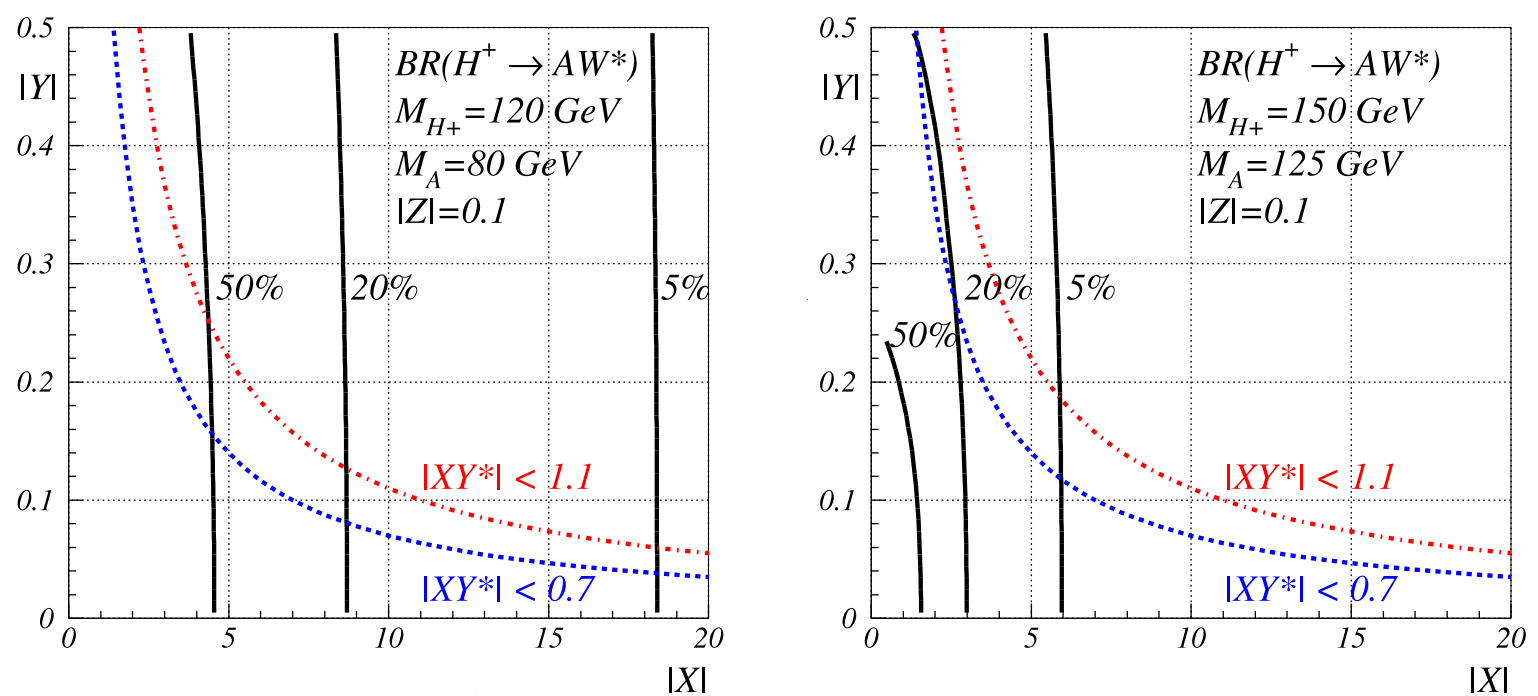

FIG. 4: Contours of $\mathrm{BR}\left(H^{ \pm} \rightarrow A^{0} W^{*}\right)$ in the plane $[X, Y]$ with $|Z|=0.1$. The constraint from $b \rightarrow s \gamma$ is shown as $\left|X Y^{*}\right|<1.1$ for $\operatorname{Re}\left(X Y^{*}\right)<0$, and $\left|X Y^{*}\right|<0.7$ for $\operatorname{Re}\left(X Y^{*}\right)>0$. In the left panel (a) we take $m_{H^{ \pm}}=120 \mathrm{GeV}$ and $m_{A^{0}}=80 \mathrm{GeV}$, and in the right panel (b) we take $m_{H^{ \pm}}=150 \mathrm{GeV}$ and $m_{A^{0}}=125 \mathrm{GeV}$. In both figures $m_{s}\left(Q=m_{H^{ \pm}}\right)=0.055 \mathrm{GeV}$.

\section{SEARCHES FOR $t \rightarrow H^{ \pm} b$ WITH $H^{ \pm} \rightarrow c s$, AND PROSPECTS FOR $H^{ \pm} \rightarrow c b$ AT THE LHC}

The case of $m_{H^{ \pm}}<m_{t}+m_{b}$ with a large $\mathrm{BR}\left(H^{ \pm} \rightarrow c s\right)$ can be searched for in the decays of the top quark via $t \rightarrow H^{ \pm} b[7,53]$. The first discussion of $t \rightarrow H^{ \pm} b$ followed by the decay $H^{ \pm} \rightarrow c b$ was given in [19]. Recently, $t \rightarrow H^{ \pm} b$ with decay $H^{ \pm} \rightarrow c b$ has been studied in the context of flipped 2HDM [21], and in the context of the 2HDM without natural flavor conservation [22].

There have been two dedicated searches by the Tevatron collaborations [12, 13] for $t \rightarrow$ 
$H^{ \pm} b$ followed by $H^{ \pm} \rightarrow c s$. The D0 analysis [12] with $1 \mathrm{fb}^{-1}$ performed a search for $t \rightarrow H^{ \pm} b$ by studying the effect of the decay $H^{ \pm} \rightarrow c s$ on ratios of cross sections for $t \bar{t}$ production. In the SM one has $\mathrm{BR}(t \rightarrow W b)=100 \%$, and the branching ratios of $W \rightarrow \ell \nu$ and $W \rightarrow q^{\prime} \bar{q}$ are known. The presence of a sizeable $\operatorname{BR}\left(t \rightarrow H^{ \pm} b\right)$ with $H^{ \pm} \rightarrow c s$ would change the $\mathrm{SM}$ prediction for the ratio of the cross sections for the channels with decay $W \rightarrow \ell \nu$ and $W \rightarrow q^{\prime} \bar{q}$. For the optimum case of $\mathrm{BR}\left(H^{ \pm} \rightarrow c s\right)=100 \%$, upper bounds on $\mathrm{BR}\left(t \rightarrow H^{ \pm} b\right)$ between 0.19 and 0.22 were obtained for $80 \mathrm{GeV}<m_{H^{ \pm}}<155 \mathrm{GeV}$. Although the decay $H^{ \pm} \rightarrow c s$ was assumed in [12] the above limits also apply (to a very good approximation) to the case of both $H^{ \pm} \rightarrow c s$ and $H^{ \pm} \rightarrow c b$ having sizeable BRs, as discussed in [21]. This is because the search strategy merely requires that $H^{ \pm}$decays to quark jets.

An alternative strategy was adopted in the CDF analysis [13] with $2.2 \mathrm{fb}^{-1}$. A direct search for the decay $H^{ \pm} \rightarrow c s$ was performed by looking for a peak centered at $M_{H^{ \pm}}$in the dijet invariant mass distribution, which would be distinct from the peak at $M_{W}$ from the SM decay $t \rightarrow W b$ with $W \rightarrow q^{\prime} \bar{q}$. For the optimum case of $\operatorname{BR}\left(H^{ \pm} \rightarrow q^{\prime} \bar{q}\right)=100 \%$, upper bounds on $\mathrm{BR}\left(t \rightarrow H^{ \pm} b\right)$ between 0.32 and 0.08 were obtained for $90 \mathrm{GeV}<m_{H^{ \pm}}<150$ $\mathrm{GeV}$, with the greatest sensitivity being at $m_{H^{ \pm}}=130 \mathrm{GeV}$. No limits on $\mathrm{BR}\left(t \rightarrow H^{ \pm} b\right)$ were given for the region $70 \mathrm{GeV}<m_{H^{ \pm}}<90 \mathrm{GeV}$ due to the large background from $W \rightarrow q^{\prime} \bar{q}$ decays. For the region $60 \mathrm{GeV}<m_{H^{ \pm}}<70 \mathrm{GeV}$, limits on $\mathrm{BR}\left(t \rightarrow H^{ \pm} b\right)$ between 0.09 and 0.12 were derived. As stated in [13], the above limits also apply to other hadronic decays of $H^{ \pm}$, although with slight changes in the sensitivity to $\operatorname{BR}\left(t \rightarrow H^{ \pm} b\right)$ because the dijet mass resolution depends mildly on the flavour of the quarks. The search strategy in [13] and does not have sensitivity to the region $80 \mathrm{GeV}<m_{H^{ \pm}}<90 \mathrm{GeV}$ due to the large background from $W \rightarrow c s, u d$. The combination of the four searches at LEP for $e^{+} e^{-} \rightarrow H^{+} H^{-} 54$ derived the limit $m_{H^{ \pm}}>81 \mathrm{GeV}$ for the scenario of $\mathrm{BR}\left(H^{ \pm} \rightarrow c s\right) \sim 100 \%$, with the following additional small intervals excluded (at 95\% c.l): $86 \mathrm{GeV}<m_{H^{ \pm}}<88 \mathrm{GeV}$ and $m_{H^{ \pm}} \sim 84 \mathrm{GeV}$. Therefore the region of $80 \mathrm{GeV}<m_{H^{ \pm}}<90 \mathrm{GeV}$ with $H^{ \pm}$decaying dominantly to quarks (e.g. $c s, c b$ ) has not yet been entirely excluded yet.

Concerning the prospects at the LHC, there has been a simulation of $t \rightarrow H^{ \pm} b$ followed by the decay $H^{ \pm} \rightarrow c s$ by the ATLAS collaboration in [55], assuming that one of the top/antitop quarks in the $t \bar{t}$ events decays leptonically via $t \rightarrow W b \rightarrow \ell \nu b$. This strategy is very similar to the CDF analysis of [13], and directly looks for a peak centered at $m_{H^{ \pm}}$ in the invariant mass distribution of the jets from $H^{ \pm} \rightarrow c s$. Two b-tags are applied, and the peak from $H^{ \pm} \rightarrow c s$ is obtained by reconstructing the two untagged jets. The mass resolution of the peak can be further improved by full reconstruction of the $t \bar{t}$ event. For $\sqrt{s}=7 \mathrm{TeV}$ with $1 \mathrm{fb}^{-1}$ of luminosity, values of $\mathrm{BR}\left(t \rightarrow H^{ \pm} b\right)$ as low as 0.04 can be probed for $110 \mathrm{GeV}<m_{H^{ \pm}}<150 \mathrm{GeV}$. This sensitivity is superior to that achieved for the decay $t \rightarrow H^{ \pm} b$ followed by $H^{ \pm} \rightarrow \tau \nu$ with the same integrated luminosity [13]. Again, as in [13] there is little or no sensitivity to the region $80 \mathrm{GeV}<m_{H^{ \pm}}<90 \mathrm{GeV}$.

The first search for $t \rightarrow H^{ \pm} b$ followed by the decay $H^{ \pm} \rightarrow c s$ at the LHC has been performed by the ATLAS collaboration with $0.035 \mathrm{fb}^{-1}$ in [14. Due to the small amount of integrated luminosity, only one $b$-tag was applied. The limits on $\operatorname{BR}\left(t \rightarrow H^{ \pm} b\right)$ are comparable to those from the Tevatron search in [13], with limits of $\operatorname{BR}\left(t \rightarrow H^{ \pm} b\right)<$ $0.25,0.15$ and 0.14 for $m_{H^{ \pm}}=90 \mathrm{GeV}, 110 \mathrm{GeV}$ and $130 \mathrm{GeV}$ respectively.

If $\operatorname{BR}\left(H^{ \pm} \rightarrow c b\right)$ were the dominant decay channel, as can be the case in the MHDM and the A2HDM, the requirement of tagging the $b$ from $H^{ \pm} \rightarrow c b$ (as suggested in [19, 21, 22]) would provide sensitivity to $\mathrm{BR}\left(t \rightarrow H^{ \pm} b\right)$ in the problematic region $80 \mathrm{GeV}<m_{H^{ \pm}}<90$ $\mathrm{GeV}$, and should improve the sensitivity for $m_{H^{ \pm}}>90 \mathrm{GeV}$. We now estimate the gain in 
sensitivity using realistic values for the $b$-tagging efficiency $\left(\epsilon_{b}=0.5\right)$, the probability of a $c$-quark being misidentified as a $b$ quark $\left(\epsilon_{c}=0.1\right)$ and the probability of a light quark being misidentified as a $b$-quark $\left(\epsilon_{j}=0.01\right)$. The two dominant backgrounds to the peak at $m_{H^{ \pm}}$ in the dijet invariant mass distribution are from $W \rightarrow u d$ and $W \rightarrow c s$, which we take to be equal in magnitude. For the case of $\mathrm{BR}\left(H^{ \pm} \rightarrow c b\right)$ near $80 \%$, the ratio of the signal to the background $(S / \sqrt{B})$ with and without the $b$-tag is given approximately as follows:

$$
\frac{[S / \sqrt{B}]_{\text {btag }}}{[S / \sqrt{B}]_{\text {btag }}} \sim \frac{\epsilon_{b} \sqrt{2}}{\sqrt{\left(\epsilon_{j}+\epsilon_{c}\right)}} \sim 2.13 .
$$

We encourage a detailed simulation by the Tevatron and LHC collaborations in order to obtain a more realistic estimate of the increase in sensitivity over the current strategy of not applying a $b$-tag to the jets originating from $H^{ \pm}$.

We note that a recent paper [56] has performed a simulation for a very similar signature which arises from the decay $t \rightarrow h^{0} c \rightarrow b \bar{b} c$ in a different 2HDM with FCNCs. This signature looks identical to the signature arising from $H^{ \pm} \rightarrow c b$ but there are several kinematical differences. The process $t \rightarrow H^{ \pm} b \rightarrow b \bar{b} c$ would give a peak at $m_{H^{ \pm}}$in the dijet invariant mass distribution in which only one of jets has originated from a $b$ quark, with the other two $b$-jets coming from the decay of $t \bar{t}$. In contrast, for $t \rightarrow h^{0} c \rightarrow b \bar{b} c$ both of jets in the dijet invariant mass distribution would originate from $b$ quarks, while the third $b$-jet would come from the decay of $t$ or $\bar{t}$. The study in [56] is specifically for $t \rightarrow h^{0} c \rightarrow b \bar{b} c$, and it was found that the sensitivity to $\operatorname{BR}\left(t \rightarrow h^{0} c\right)$ was significantly superior to that for $t \rightarrow H^{ \pm} b$ followed by $H^{ \pm} \rightarrow c s$, which can be attributed to the extra $b$-tag i.e. the increase in sensitivity in [56] compared to that obtained for the LHC simulation without the $b$-tag in [55] is significantly greater than the value of 2.13 in eq. (12), and could be as large as a factor of six.

\section{NUMERICAL RESULTS}

We now quantify the magnitude of $H^{ \pm} \rightarrow c b$ events produced in the decays of $t$ quarks, and compare this with the expected sensitivity at the LHC. For the partial decay widths of $t \rightarrow W^{ \pm} b$ and $t \rightarrow H^{ \pm} b$ we use the leading-order expressions (with $\left|V_{t b}\right|=1$ ) as follows:

$$
\begin{array}{r}
\Gamma\left(t \rightarrow W^{ \pm} b\right)=\frac{G_{F} m_{t}}{8 \sqrt{2} \pi}\left[m_{t}^{2}+2 M_{W}^{2}\right]\left[1-M_{W}^{2} / m_{t}^{2}\right]^{2} \\
\Gamma\left(t \rightarrow H^{ \pm} b\right)=\frac{G_{F} m_{t}}{8 \sqrt{2} \pi}\left[m_{t}^{2}|Y|^{2}+m_{b}^{2}|X|^{2}\right]\left[1-m_{H^{ \pm}}^{2} / m_{t}^{2}\right]^{2}
\end{array}
$$

The multiplicative (vertex) QCD corrections to both $t \rightarrow W^{ \pm} b$ and $t \rightarrow H^{ \pm} b$ essentially cancel out in the ratio of partial widths [57], and thus they do not affect $\operatorname{BR}\left(t \rightarrow H^{ \pm} b\right)$ significantly. In the phase-space function of both decays we neglect $m_{b}$, and in the terms $m_{t}^{2}|Y|^{2}$ and $m_{b}^{2}|X|^{2}$ we use $m_{t}=175 \mathrm{GeV}$ and $m_{b}$ evaluated at the scale of $m_{H^{ \pm}}$(i.e. $\left.m_{b} \sim 2.95 \mathrm{GeV}\right)$.

In Fig. (5a) and Fig. (5b) we show contours of the sum of

$$
\mathrm{BR}\left(t \rightarrow H^{ \pm} b\right) \times\left[\mathrm{BR}\left(H^{ \pm} \rightarrow c s\right)+\mathrm{BR}\left(H^{ \pm} \rightarrow c b\right)\right]
$$

in the plane of $[X, Y]$ for $m_{H^{ \pm}}=80 \mathrm{GeV}$ and $m_{H^{ \pm}}=120 \mathrm{GeV}$ respectively, setting $|Z|=0.1$. The cross section in eq. (14) is the signature to which the current search strategy at the 
Tevatron and the LHC is sensitive, i.e. one $b$-tag (LHC [14]) or two $b$-tags (Tevatron [13]) are applied to the jets originating from $t \bar{t}$ decay, but no $b$-tag is applied to the jets originating from $H^{ \pm}$. For the case of $\left[\mathrm{BR}\left(H^{ \pm} \rightarrow c s\right)+\mathrm{BR}\left(H^{ \pm} \rightarrow c b\right)\right]=100 \%$ the current experimental limits for $m_{H^{ \pm}}=120 \mathrm{GeV}$ are $\mathrm{BR}\left(t \rightarrow H^{ \pm} b\right)<0.14$ from ATLAS with $0.035 \mathrm{fb}^{-1}$ [14], $\mathrm{BR}\left(t \rightarrow H^{ \pm} b\right)<0.12$ from $\mathrm{CDF}$ with $2.2 \mathrm{fb}^{-1}[13]$, and $\mathrm{BR}\left(t \rightarrow H^{ \pm} b\right)<0.22$ from D0 with $1 \mathrm{fb}^{-1}[12]$. In Fig. (5b) for $m_{H^{ \pm}}=120 \mathrm{GeV}$ these upper limits would exclude the parameter space of $|X|>40$ and small $|Y|$ which is not excluded by the constraint from $b \rightarrow s \gamma$. For the mass region $80 \mathrm{GeV}<m_{H^{ \pm}}<90 \mathrm{GeV}$ there is only a limit from the D0 search in [12], which gives $\mathrm{BR}\left(t \rightarrow H^{ \pm} b\right)<0.21$. From Fig. (5a), for $m_{H^{ \pm}}=80 \mathrm{GeV}$ one can see that this limit excludes the parameter space of $|X|>35$ and small $|Y|$.

In both Fig. (5a) and Fig. (15b) we show contours of $1 \%$, which might be reachable in the $8 \mathrm{TeV}$ run of the LHC. Simulations by ATLAS (with $\sqrt{s}=7 \mathrm{TeV}$ ) for $H^{ \pm} \rightarrow$ cs [55] have shown that the LHC should be able to probe values $\operatorname{BR}\left(t \rightarrow H^{ \pm} b\right)>0.05$ with $1 \mathrm{fb}^{-1}$ for $m_{H^{ \pm}}>110 \mathrm{GeV}$, with the greatest sensitivity being around $m_{H^{ \pm}}=130 \mathrm{GeV}$. For the operation with $\sqrt{s}=8 \mathrm{TeV}$ and an anticipated integrated luminosity of $15 \mathrm{fb}^{-1}$ one expects increased sensitivity (e.g. $\mathrm{BR}\left(t \rightarrow H^{ \pm} b\right)>0.01$ for $m_{H^{ \pm}}>110 \mathrm{GeV}$ ), although the region $80 \mathrm{GeV}<m_{H^{ \pm}}<90 \mathrm{GeV}$ might remain difficult to probe with the strategy of reconstructing the jets from $H^{ \pm}$. An alternative way to probe the region $80 \mathrm{GeV}<m_{H^{ \pm}}<90 \mathrm{GeV}$ is to use the search strategy by D0 in [12], and presumably the LHC could improve on the Tevatron limit on $\mathrm{BR}\left(t \rightarrow H^{ \pm} b\right)<0.21$ for $80 \mathrm{GeV}<m_{H^{ \pm}}<90 \mathrm{GeV}$. From Fig. (5) ) (for $m_{H^{ \pm}}=120$ $\mathrm{GeV}$ ) one can see that the region of $|Y|>0.2$ and $|X|<4$, which is not excluded by $b \rightarrow s \gamma$, would be probed if sensitivity to $\operatorname{BR}\left(t \rightarrow H^{ \pm} b\right)>0.01$ were achieved. However, a large part of the region roughly corresponding to $|Y|<0.2$ and $|X|<10$ (which is also not excluded by $b \rightarrow s \gamma)$ would require sensitivity to $\mathrm{BR}\left(t \rightarrow H^{ \pm} b\right)<0.01$ in order to be probed with the current search strategy for $t \rightarrow H^{ \pm} b$, and this is probably unlikely in the $8 \mathrm{TeV}$ run of the LHC.

Increased sensitivity to the plane of $[X, Y]$ can be achieved by requiring a $b$-tag on the jets which originate from the decay of $H^{ \pm}$. In Figs. (6) and (7), for $m_{H^{ \pm}}=80 \mathrm{GeV}$ and $m_{H^{ \pm}}=120 \mathrm{GeV}$ respectively, we show contours of

$$
\mathrm{BR}\left(t \rightarrow H^{ \pm} b\right) \times \mathrm{BR}\left(H^{ \pm} \rightarrow c b\right) .
$$

With the extra $b$-tag, as described in eq. (12), the sensitivity should reach $\mathrm{BR}(t \rightarrow$ $\left.H^{ \pm} b\right) \times \operatorname{BR}\left(H^{ \pm} \rightarrow c b\right)>0.5 \%$, and perhaps as low as $0.2 \%$. In the latter case, one can see from Figs. (6) and (7) that a large part of the regions of $|X|<5$ (for $m_{H^{ \pm}}=120 \mathrm{GeV}$ ) and $|X|<3$ (for $m_{H^{ \pm}}=80 \mathrm{GeV}$ ) could be probed, even for $|Y|<0.2$. Therefore there would be sensitivity to a sizeable region of the parameter space of $[X, Y]$ which is not excluded by $b \rightarrow s \gamma$, a result which is in contrast to the above case where no $b$-tag is applied to the $b$-jets originating from $H^{ \pm}$. We encourage a dedicated search for $t \rightarrow H^{ \pm} b$ and $H^{ \pm} \rightarrow c b$ by the Tevatron and LHC collaborations. Such a search would be a well-motivated extension and application of the searches which have already been carried out in [13] and [14], and would offer the possibility of increased sensitivity to the fermionic couplings and mass of $H^{ \pm}$in the A2HDM and a MHDM.

\section{CONCLUSIONS}

Light charged Higgs bosons $\left(H^{ \pm}\right)$are being searched for in the decays of top quarks $\left(t \rightarrow H^{ \pm} b\right)$ at the Tevatron and at the LHC. Separate searches are being carried out for 

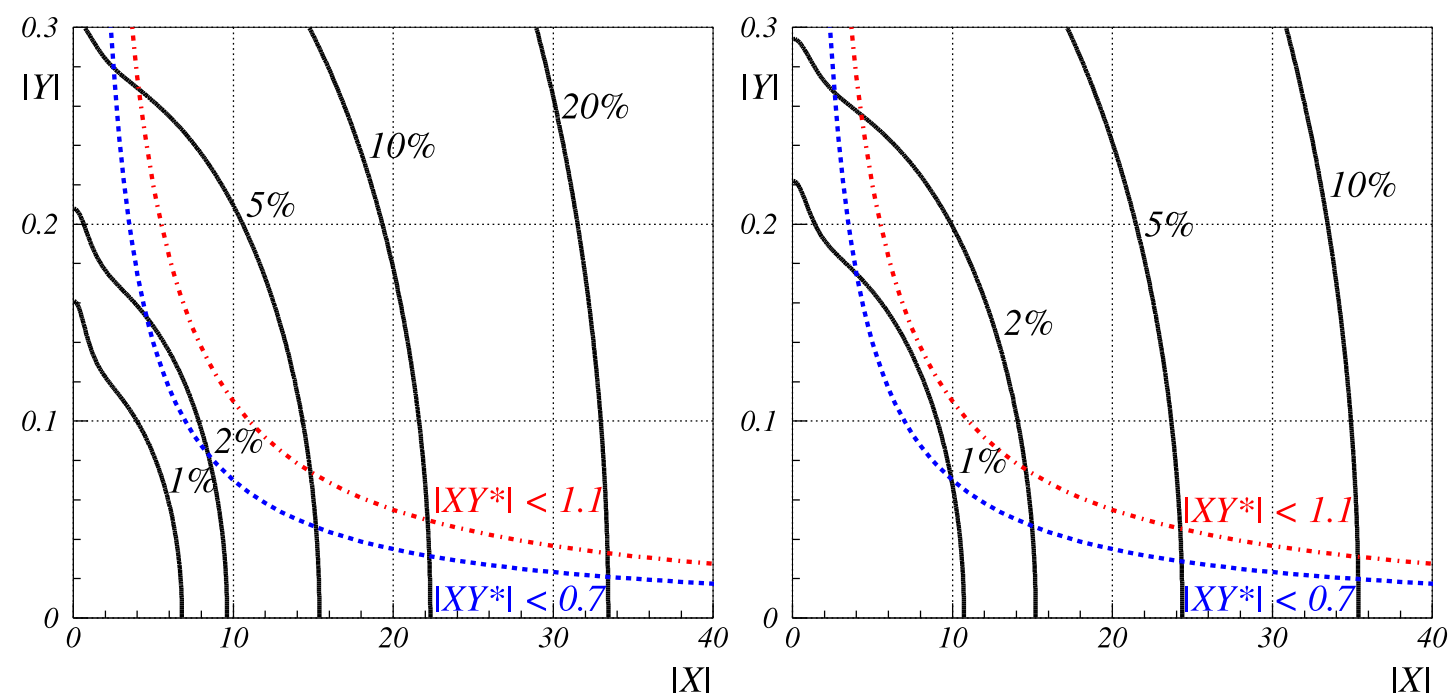

FIG. 5: Contours of the sum of $\mathrm{BR}\left(t \rightarrow H^{ \pm} b\right) \times \mathrm{BR}\left(H^{ \pm} \rightarrow c s\right)$ and $\mathrm{BR}\left(t \rightarrow H^{ \pm} b\right) \times \mathrm{BR}\left(H^{ \pm} \rightarrow c b\right)$ in the plane $[X, Y]$ with $|Z|=0.1$, where $m_{H^{ \pm}}=80 \mathrm{GeV}$ (left panel) and $m_{H^{ \pm}}=120 \mathrm{GeV}$ (right panel).
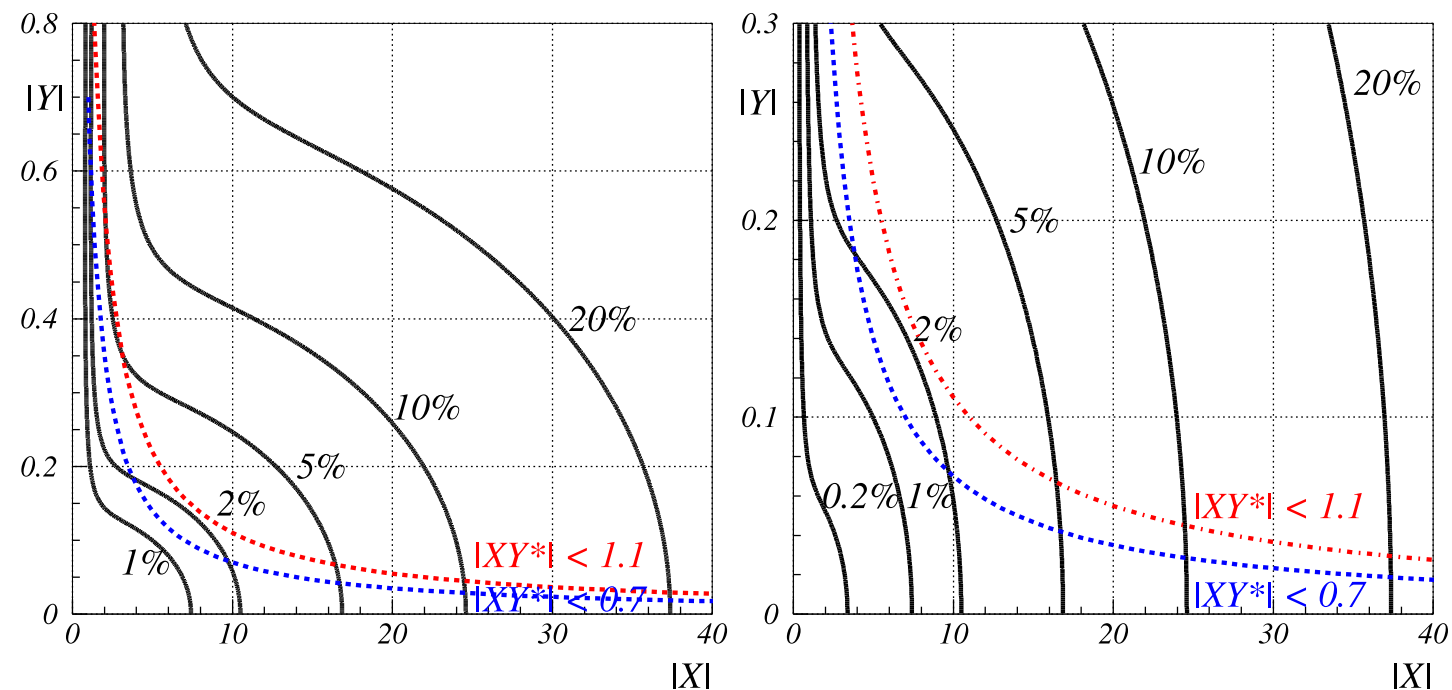

FIG. 6: Contours of $\mathrm{BR}\left(t \rightarrow H^{ \pm} b\right) \times \mathrm{BR}\left(H^{ \pm} \rightarrow c b\right)$ in the plane $[X, Y]$ with $|Z|=0.1$ for $m_{H^{ \pm}}=80$ $\mathrm{GeV}$. The constraint from $b \rightarrow s \gamma$ is shown as $\left|X Y^{*}\right|<1.1$ for $\operatorname{Re}\left(X Y^{*}\right)<0$, and $\left|X Y^{*}\right|<0.7$ for $\operatorname{Re}\left(X Y^{*}\right)>0$. We take $m_{s}\left(Q=m_{H^{ \pm}}\right)=0.055 \mathrm{GeV}$ and show the range $0<|Y|<0.8$ (left panel) and $0<|Y|<0.3$ (right panel).

the decay channels $H^{ \pm} \rightarrow c s$ and $H^{ \pm} \rightarrow \tau \nu$, with comparable sensitivity to the mass and fermionic couplings of $H^{ \pm}$. The searches for $H^{ \pm} \rightarrow c s$ in [13] and [14] look for a peak at $m_{H^{ \pm}}$in the dijet invariant mass distribution, with the assumption that neither of the quarks is a $b$ quark.

In some models with two or more Higgs doublets (the Aligned 2HDM and a MHDM with three or more scalar doublets) the branching ratio for $H^{ \pm} \rightarrow c b$ can be as large as $80 \%$. 

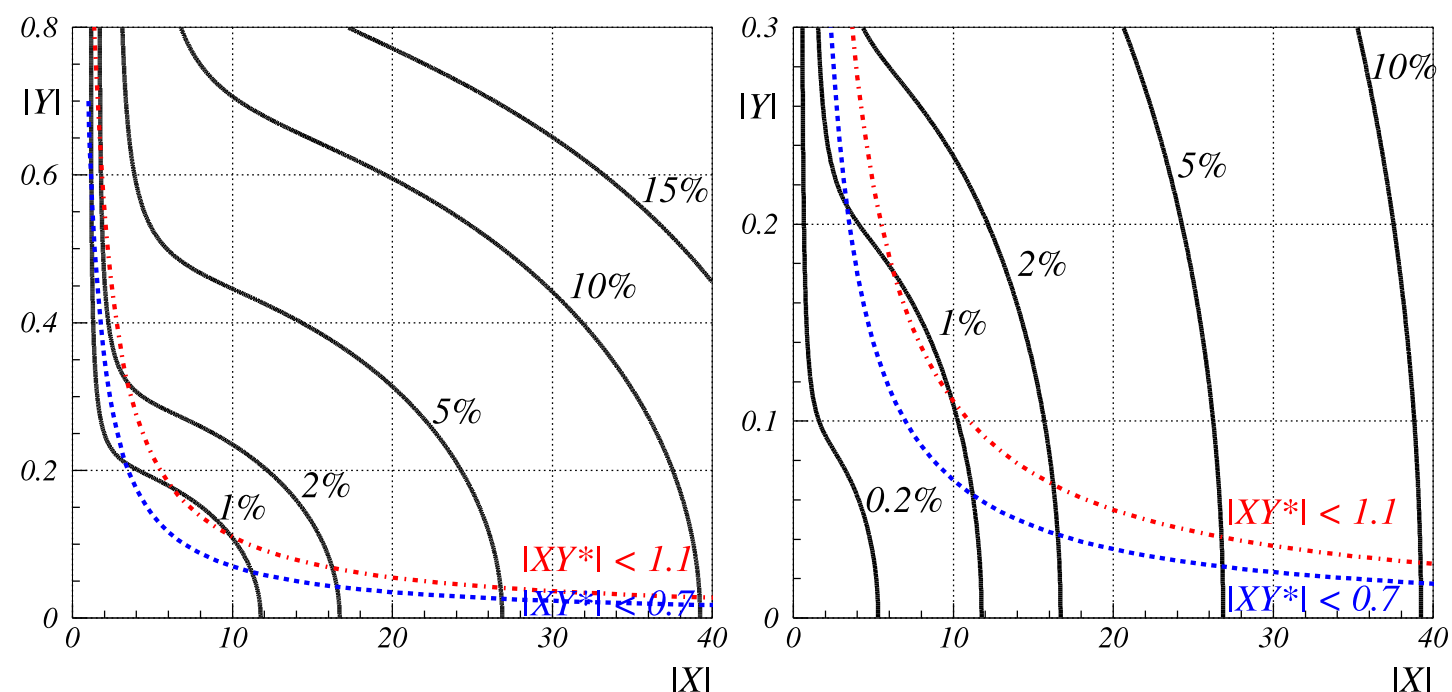

FIG. 7: Contours of $\operatorname{BR}\left(t \rightarrow H^{ \pm} b\right) \times \mathrm{BR}\left(H^{ \pm} \rightarrow c b\right)$ in the plane $[X, Y]$ with $|Z|=0.1$ for $m_{H^{ \pm}}=120 \mathrm{GeV}$. The constraint from $b \rightarrow s \gamma$ is shown as $\left|X Y^{*}\right|<1.1$ for $\operatorname{Re}\left(X Y^{*}\right)<0$, and $\left|X Y^{*}\right|<0.7$ for $\operatorname{Re}\left(X Y^{*}\right)>0$. We take $m_{s}\left(Q=m_{H^{ \pm}}\right)=0.055 \mathrm{GeV}$ and show the range $0<|Y|<0.8$ (left panel) and $0<|Y|<0.3$ (right panel).

Moreover, such a $H^{ \pm}$could be light enough to be produced via $t \rightarrow H^{ \pm} b$, as well as respect the stringent constraints from $b \rightarrow s \gamma$ on both $m_{H^{ \pm}}$and the fermionic couplings of $H^{ \pm}$. This is in contrast to $H^{ \pm}$in other 2HDMs for which a large branching ratio for $H^{ \pm} \rightarrow c b$ is possible (such as the flipped 2HDM for $m_{H^{ \pm}}<m_{t}$ ), but one expects $m_{H^{ \pm}}>m_{t}$ in order to comply with the measured value of $b \rightarrow s \gamma$. In the context of the Aligned 2HDM and a MHDM we suggested that a dedicated search for $t \rightarrow H^{ \pm} b$ and $H^{ \pm} \rightarrow c b$ would probe values of the fermionic couplings of $H^{ \pm}$which are currently not excluded by measurements of $b \rightarrow s \gamma$. Such a search would require a $b$-tag of one of the jets originating from $H^{ \pm}$, and would afford sensitivity to a smaller value of the branching ratio of $t \rightarrow H^{ \pm} b$ than that obtained in the ongoing searches, which currently do not make use of this additional $b$-tag. We emphasised that a dedicated search for $t \rightarrow H^{ \pm} b$ and $H^{ \pm} \rightarrow c b$ at the Tevatron and LHC would be a well-motivated and (possibly) straightforward extension of the ongoing searches for $t \rightarrow H^{ \pm} b$ with decay $H^{ \pm} \rightarrow c s$.

\section{Acknowledgements}

A.G.A was supported by a Marie Curie Incoming International Fellowship, FP7PEOPLE-2009-IIF, Contract No. 252263. J. H.-S acknowledges the financial support of SNI, PROMEP and VIEP-BUAP. S.M is supported in part by the NExT Institute. J. H.-S. thanks the University of Southampton and the Rutherford Appleton Laboratory for hospitality during his visit to the NExT Institute where part of this work was carried out. Useful 
comments from A. Stuart and A. Lytle are gratefully acknowledged.

[1] G. Aad et al. [ATLAS Collaboration], Phys. Lett. B 710, 49 (2012).

[2] S. Chatrchyan et al. [CMS Collaboration], arXiv:1202.1488 [hep-ex].

[3] S. Chatrchyan et al. [CMS Collaboration], CMS PAS HIG-12-008; G. Aad et al. [ATLAS Collaboration], ATLAS-CONF-2012-019.

[4] T. D. Lee, Phys. Rev. D 8, 1226 (1973); H. E. Haber, G. L. Kane and T. Sterling, Nucl. Phys. B 161, 493 (1979); J. F. Gunion, H. E. Haber, G. L. Kane and S. Dawson, "The Higgs Hunter's Guide," Front. Phys. 80, 1 (2000).

[5] G. C. Branco, P. M. Ferreira, L. Lavoura, M. N. Rebelo, M. Sher and J. P. Silva, arXiv:1106.0034 [hep-ph] (to appear in Physics Reports).

[6] S. L. Glashow and S. Weinberg, Phys. Rev. D 15, 1958 (1977); E. A. Paschos, Phys. Rev. D 15, 1966 (1977).

[7] V. D. Barger, J. L. Hewett and R. J. N. Phillips, Phys. Rev. D 41, 3421 (1990).

[8] D. P. Roy, Mod. Phys. Lett. A 19, 1813 (2004); A. Djouadi, Phys. Rept. 459, 1 (2008).

[9] D. Eriksson, F. Mahmoudi and O. Stal, JHEP 0811, 035 (2008); F. Mahmoudi and O. Stal, Phys. Rev. D 81, 035016 (2010).

[10] M. Aoki, R. Guedes, S. Kanemura, S. Moretti, R. Santos and K. Yagyu, Phys. Rev. D 84, 055028 (2011).

[11] J. F. Gunion, H. E. Haber, F. E. Paige, W. K. Tung and S. S. D. Willenbrock, Nucl. Phys. B 294, 621 (1987); J. L. Diaz-Cruz and O. A. Sampayo, Phys. Rev. D 50, 6820 (1994); S. Moretti and D. P. Roy, Phys. Lett. B 470, 209 (1999); D. J. Miller, S. Moretti, D. P. Roy and W. J. Stirling, Phys. Rev. D 61, 055011 (2000).

[12] V. M. Abazov et al. [D0 Collaboration], Phys. Lett. B 682, 278 (2009).

[13] T. Aaltonen et al. [CDF Collaboration], Phys. Rev. Lett. 103, 101803 (2009).

[14] G. Aad et al. [ATLAS Collaboration] ATLAS-CONF-2011-094, (July 2011).

[15] G. Aad et al. [ATLAS Collaboration] ATLAS-CONF-2012-011 (March 2012).

[16] S. Chatrchyan et al. [CMS Collaboration] CMS-PAS-HIG-11-008 (July 2011).

[17] Y. Grossman, Nucl. Phys. B 426, 355 (1994).

[18] A. G. Akeroyd and W. J. Stirling, Nucl. Phys. B 447, 3 (1995).

[19] A. G. Akeroyd, arXiv:hep-ph/9509203.

[20] A. G. Akeroyd, Nucl. Phys. B 544, 557 (1999).

[21] H. E. Logan and D. MacLennan, Phys. Rev. D 81, 075016 (2010).

[22] J. L. Diaz-Cruz, J. Hernandez-Sanchez, S. Moretti, R. Noriega-Papaqui and A. Rosado, Phys. Rev. D 79, 095025 (2009).

[23] M. Aoki, S. Kanemura, K. Tsumura and K. Yagyu, Phys. Rev. D 80, 015017 (2009).

[24] W. S. Hou and R. S. Willey, Phys. Lett. B 202, 591 (1988); T. G. Rizzo, Phys. Rev. D 38, 820 (1988); B. Grinstein, R. P. Springer and M. B. Wise, Phys. Lett. B 202, 138 (1988); B. Grinstein, R. P. Springer and M. B. Wise, Nucl. Phys. B 339, 269 (1990).

[25] F. Borzumati and C. Greub, Phys. Rev. D 58, 074004 (1998); F. Borzumati and C. Greub, Phys. Rev. D 59, 057501 (1999).

[26] M. Misiak, H. M. Asatrian, K. Bieri, M. Czakon, A. Czarnecki, T. Ewerth, A. Ferroglia and P. Gambino et al., Phys. Rev. Lett. 98, 022002 (2007).

[27] A. Pich and P. Tuzon, Phys. Rev. D 80, 091702 (2009). 
[28] M. Jung, A. Pich and P. Tuzon, JHEP 1011, 003 (2010).

[29] M. Trott and M. B. Wise, JHEP 1011, 157 (2010).

[30] G. Cree and H. E. Logan, Phys. Rev. D 84, 055021 (2011).

[31] T. P. Cheng and M. Sher, Phys. Rev. D 35, 3484 (1987).

[32] R. M. Barnett, G. Senjanovic, L. Wolfenstein and D. Wyler, Phys. Lett. B 136, 191 (1984);

R. M. Barnett, G. Senjanovic and D. Wyler, Phys. Rev. D 30, 1529 (1984).

[33] S. Su and B. Thomas, Phys. Rev. D 79, 095014 (2009).

[34] V. Barger, H. E. Logan and G. Shaughnessy, Phys. Rev. D 79, 115018 (2009).

[35] A. G. Akeroyd, Phys. Lett. B 377, 95 (1996); A. G. Akeroyd, J. Phys. G 24, 1983 (1998).

[36] S. Durr, Z. Fodor, C. Hoelbling, S. D. Katz, S. Krieg, T. Kurth, L. Lellouch and T. Lippert et al., Phys. Lett. B 701, 265 (2011); C. McNeile, C. T. H. Davies, E. Follana, K. Hornbostel and G. P. Lepage, Phys. Rev. D 82, 034512 (2010); J. Laiho and R. S. Van de Water, arXiv:1112.4861 [hep-lat]; A. Bazavov et al. [The MILC Collaboration], PoS LAT 2009, 079 (2009); T. Blum, R. Zhou, T. Doi, M. Hayakawa, T. Izubuchi, S. Uno and N. Yamada, Phys. Rev. D 82, 094508 (2010); C. Kelly, arXiv:1201.0706 [hep-lat].

[37] J. Laiho, E. Lunghi and R. S. Van de Water, Phys. Rev. D 81, 034503 (2010). http://mypage.iu.edu/ elunghi/webpage/LatAves/index.html

[38] G. Colangelo, S. Durr, A. Juttner, L. Lellouch, H. Leutwyler, V. Lubicz, S. Necco and C. T. Sachrajda et al., Eur. Phys. J. C 71, 1695 (2011).

[39] K. Nakamura et al. [Particle Data Group Collaboration], J. Phys. G 37, 075021 (2010).

[40] S. Moretti and W. J. Stirling, Phys. Lett. B 347, 291 (1995) [Erratum-ibid. B 366, 451 (1996)]; A. Djouadi, J. Kalinowski and P. M. Zerwas, Z. Phys. C 70, 435 (1996).

[41] J. Abdallah et al. [DELPHI Collaboration], Eur. Phys. J. C 34, 399 (2004); G. Abbiendi et al. [OPAL Collaboration], arXiv:0812.0267 [hep-ex].

[42] D. Toussaint, Phys. Rev. D 18, 1626 (1978); S. Bertolini, Nucl. Phys. B 272, 77 (1986).

[43] M. E. Peskin and T. Takeuchi, Phys. Rev. Lett. 65, 964 (1990); M. E. Peskin and T. Takeuchi, Phys. Rev. D 46, 381 (1992).

[44] S. Kanemura, Y. Okada, H. Taniguchi and K. Tsumura, Phys. Lett. B 704, 303 (2011).

[45] P. H. Chankowski, M. Krawczyk and J. Zochowski, Eur. Phys. J. C 11, 661 (1999); A. Wahab El Kaffas, P. Osland and O. M. Ogreid, Phys. Rev. D 76, 095001 (2007).

[46] W. Grimus, L. Lavoura, O. M. Ogreid and P. Osland, Nucl. Phys. B 801, 81 (2008).

[47] CDF Collaboration, CDF Note 10104 (June 2010).

[48] R. Dermisek and J. F. Gunion, Phys. Rev. D 79, 055014 (2009).

[49] G. Burdman, C. Haluch and R. Matheus, arXiv:1112.3961 [hep-ph].

[50] P. M. Ferreira, R. Santos, M. Sher and J. P. Silva, arXiv:1112.3277 [hep-ph]; A. Arhrib, R. Benbrik and N. Gaur, arXiv:1201.2644 [hep-ph].

[51] D. Carmi, A. Falkowski, E. Kuflik and T. Volansky, arXiv:1202.3144 [hep-ph]; A. Azatov, R. Contino and J. Galloway, JHEP 1204, 127 (2012); J. Ellis and T. You, arXiv:1204.0464 [hep-ph].

[52] E. Gabrielli, B. Mele and M. Raidal, arXiv:1202.1796 [hep-ph]; P. P. Giardino, K. Kannike, M. Raidal and A. Strumia, arXiv:1203.4254 [hep-ph].

[53] R. M. Barnett, R. Cruz, J. F. Gunion and B. Hubbard, Phys. Rev. D 47, 1048 (1993).

[54] [LEP Higgs Working Group for Higgs boson searches and ALEPH and DELPHI and L3 and OPAL Collaborations], hep-ex/0107031.

[55] A. Ferrari [ATLAS Collaboration], PoS CHARGED2010, 010 (2010); ATL-PHYS-PUB2010-009. 
[56] C. Kao, H. -Y. Cheng, W. -S. Hou and J. Sayre, arXiv:1112.1707 [hep-ph].

[57] C. S. Li and T. C. Yuan, Phys. Rev. D 42, 3088 (1990) [Erratum-ibid. D 47, 2156 (1993)];

A. Czarnecki and S. Davidson, Phys. Rev. D 48, 4183 (1993). 